\title{
Anterior cingulate cortex abnormalities associated with a first psychotic episode in bipolar disorder
}

Alex Fornito, Murat Yücel, Stephen J. Wood, Andreas Bechdolf, Simon Carter, Chris Adamson, Dennis Velakoulis, Michael M. Saling, Patrick D. McGorry and Christos Pantelis

\section{Background}

The anterior cingulate cortex is frequently implicated in the pathophysiology of bipolar disorder, but magnetic resonance imaging (MRI) studies have reported variable findings owing to a reliance on patient samples with chronic illness and to limited appreciation of the region's heterogeneity.

\section{Aims}

To characterise anterior cingulate cortex abnormalities in patients with bipolar disorder experiencing their first episode of psychosis while accounting for regional anatomical variability.

\section{Method}

Grey matter volume, surface area and cortical thickness were measured in six anterior cingulate cortex subregions per hemisphere using MRI scans acquired from 26 patients with bipolar I disorder experiencing first-episode psychosis and 26 healthy controls matched for age, gender and regional morphological variability.

\section{Results}

Relative to controls, male patients displayed increased thickness in the right subcallosal limbic anterior cingulate cortex. No significant differences were identified in females for grey matter volume or surface area measures. The findings were not attributable to medication effects.

\section{Conclusions}

These data suggest that first-episode psychosis in bipolar disorder is associated with a gender-specific, right-lateralised thickness increase in anterior cingulate cortex subregions known to play a role in regulating physiological stress responses.

\section{Declaration of interest}

None.
The anterior cingulate cortex is a brain region critical for integrating cognitive and emotional functions in support of adaptive, goal-directed behaviour, ${ }^{1,2}$ and abnormalities in this region play a significant role in the pathophysiology of bipolar disorder. ${ }^{3}$ Early attention focused on a subcallosal region of the anterior cingulate cortex ventral to the genu (also termed subgenual prefrontal cortex), following initial magnetic resonance imaging (MRI) evidence of a left-lateralised volumetric reduction in this region in patients with chronic unipolar depression or bipolar disorder. ${ }^{4}$ However, other reports of right-sided subcallosal reductions ${ }^{5}$ or no group differences, ${ }^{6,7}$ coupled with findings of volumetric reductions in other subregions ${ }^{8,9}$ and even grey matter increases, ${ }^{10,11}$ have led to confusion regarding the precise nature of abnormalities observed in people with bipolar disorder.

One possible reason for such inconsistencies is that much of the work to date has focused on patients with chronic illness, making it difficult to determine whether the observed changes are a result of prolonged illness duration or are apparent from earlier illness stages. Studying patient samples with first-episode psychoses can help mitigate these effects, although the findings of the only four such studies that have been published have been variable: Hirayasu et $\mathrm{al}^{12}$ replicated earlier findings ${ }^{4}$ by identifying a volumetric reduction in the left subcallosal anterior cingulate cortex of patients with a positive family history; Kubicki et al ${ }^{13}$ and Farrow et $\mathrm{al}^{14}$ found no differences in grey matter using voxel-based techniques; and Adler et $a l^{11}$ found evidence of increased grey matter in caudal anterior cingulate cortex regions.

A second reason for inconsistencies in the literature is that no study to date has adequately accounted for the substantial interindividual variability in sulcal and gyral anatomy of the anterior cingulate cortex, which differs between patients with bipolar disorder and healthy populations, ${ }^{15}$ and has been shown to affect volumetric and other morphometric measures in the region. ${ }^{16-18}$ Such findings indicate that spurious differences in group comparisons might emerge if patients and controls are not well matched for sulcal anatomy. To address these limitations, we applied a novel cortical surface-based protocol for parcellating the anterior cingulate cortex into functionally relevant subdivisions while accounting for the region's anatomical variability ${ }^{17,19}$ to MRI data obtained from a sample of patients with bipolar disorder experiencing their first psychotic episode. Importantly, we individually matched patients and controls for age, gender and sulcal variability to ensure that any identified changes could not be attributed to group differences in cortical folding patterns. Our surface-based approach enabled calculation of regional grey matter volume, surface area and cortical thickness with submillimeter resolution, allowing us to detect relatively subtle changes that may be occurring in earlier stages of the disorder. Following current theories emphasising a primary role for subcallosal abnormalities in the pathophysiology of bipolar disorder, ${ }^{20}$ we expected that patients would show particularly pronounced grey matter reductions in this region.

\section{Method}

\section{Participants}

The patient sample comprised 26 patients with bipolar I disorder experiencing a first psychotic episode who were recruited from the Early Psychosis Prevention and Intervention Centre, Melbourne, Australia. All patients were in a manic state at presentation. DSM-IV ${ }^{21}$ diagnoses were confirmed over a minimum 6-month follow-up period and assigned based on medical record review and the Structured Clinical Interview for DSM-IV (SCID). ${ }^{22}$ None of the patients had a history of childhood-onset bipolar disorder. Further details regarding recruitment procedures are 
published elsewhere. ${ }^{23}$ Twelve (eight male) patients were taking atypical antipsychotics at the time of scanning and nine (five male) were taking typical agents (mean chlorpromazine equivalents at time of scanning: $188.04 \mathrm{mg} /$ day, s.d. $=119.16)$. Seven patients (four male) were taking lithium: two adjunctively with typical antipsychotics, four with atypicals and one as monotherapy. Two patients were medication-free. Treatment data were not available for two patients. All patients were experiencing psychotic symptoms at the time of scanning.

Prior psychiatric treatment in this sample was as follows: 16 none, 3 depression (all were on antidepressants at MRI; maximum treatment duration was 2 months), 1 post-traumatic stress disorder (not treated at study intake). One patient reported experiencing two manic episodes 5 years prior to the study but did not seek treatment. Family history of psychiatric illness was as follows: 11 none, 1 bipolar, 1 bipolar and schizophrenia, 1 schizophrenia, 4 depression, 2 depression plus anxiety. Clinical histories were unavailable for five patients (excluding these participants did not change the findings, so their data were retained for the final analysis).

Healthy controls with no personal history of mental or neurological illness and no family history of psychosis were selected from our larger database in order to individually match them to each patient for age, gender and anterior cingulate cortex sulcal morphology (see below). Exclusion criteria for all participants included a history of steroid misuse, substantial head injury and impaired thyroid function. Participants were also excluded if they had ever met diagnostic criteria for a substance use/dependence disorder, given evidence that such disorders can affect grey matter. ${ }^{24}$ Patients with comorbid psychiatric, neurological or significant medical conditions were also excluded from the study (see Velakoulis et $a l^{23}$ for more details). All participants gave written, informed consent in accordance with local ethics committee guidelines. Demographic details are presented in Table 1.

\section{Magnetic resonance imaging}

\section{Image acquisition}

Scans were acquired using a GE Signa $1.5 \mathrm{~T}$ scanner at the Royal Melbourne Hospital, Victoria, Australia. A three-dimensional volumetric SPGR sequence generated 124 contiguous coronal slices. Imaging parameters were: time to echo (TE), $3.3 \mathrm{~ms}$; time to repetition (TR), $14.3 \mathrm{~ms}$; flip angle, $30^{\circ}$; matrix size, $256 \times 256$; field of view, $24 \times 24 \mathrm{~cm}$; voxel dimensions, $0.938 \times 0.938 \times 1.5 \mathrm{~mm}$. Data were transferred from DAT tape to a Linux Debian 3.1 workstation and coded to ensure participant confidentiality and masked rating.

\section{Image pre-processing}

Prior to classifying sulcal morphology, each participants' image was stripped of extracerebral tissue and aligned to the N27 template via a $6^{\circ}$ rigid-body transformation using tools contained in the FSL software package (www.fmrib.ox.ac.uk/fsl). No rescaling or warping was performed, but the images were resampled to $1 \mathrm{~mm}^{3}$ voxels in the process.

\section{Classification of sulcal variability}

There are two major variations in sulcal and gyral anatomy that can affect region-of-interest boundaries and alter regional morphometry: the incidence and extent of the paracingulate sulcus, and the confluence of the cingulate sulcus with the superior rostral sulcus. ${ }^{19}$ The paracingulate sulcus runs dorsal and parallel to the cingulate sulcus in $30-60 \%$ of cases. ${ }^{25}$ We classified the paracingulate sulcus as 'present' if there was a clearly identifiable sulcus running dorsal and parallel to the cingulate sulcus that was at least $20 \mathrm{~mm}$ in length, or 'absent' if no such sulcus was apparent, according to reliable and established criteria (see Fornito et $a l^{19}$ and Yücel et $a l^{25}$ ). The superior rostral sulcus was classified as 'continuous' with the cingulate sulcus if the two were connected rostral to the genu of the corpus callosum; all other cases were classified as 'separate' (see Fornito et al ${ }^{19}$ and online Fig. DS1). All sulcal classifications were performed on the N27-aligned images using Analyze 6.0 for Linux (Mayo Software, Englewood, Colorado, USA).

Prior work has shown that the appearance of a paracingulate sulcus affects the size and location of anterior cingulate cortex subregions. When a paracingulate sulcus is present, the paralimbic anterior cingulate cortex (ACC $\mathrm{A}_{\mathrm{P}}$; primarily Brodmann area 32 ) extends from the depths of the cingulate sulcus across the crown of the paracingulate gyrus to the paracingulate sulcus; when the paracingulate sulcus is absent, the $\mathrm{ACC}_{\mathrm{P}}$ is buried in the depths of the cingulate sulcus ${ }^{19,26}$ (online Fig. DS1). Limbic ACC (ACC ; Brodmann area 24) is generally located between the callosal and cingulate sulci, although its size is also affected by paracingulate sulcus variability. ${ }^{16,17,19}$ This effect can be quite large, with one study suggesting that the appearance of a paracingulate sulcus leads to $39 \%$ decrease in ACC $_{L}$ volume and $88 \%$ increase in ACC $_{P}$ volume in some cases. ${ }^{19}$ Similar findings have been observed for regional surface area, although the relationship with cortical thickness is more complicated. ${ }^{17}$ Such findings, coupled with evidence that the paracingulate sulcus is less frequent in patients with bipolar disorder, ${ }^{15}$ indicate that spurious group differences may emerge if the comparison groups are not well matched for this morphological variation. To this end, we devised a

Table 1 Sample characteristics

\begin{tabular}{|c|c|c|c|}
\hline & Patients with bipolar disorder & Controls & $P$ \\
\hline Males/females, $n$ & $16 / 10$ & $16 / 10$ & \\
\hline Right/left/mixed-handed, $n$ & $22 / 3 / 1$ & $23 / 3 / 0$ & \\
\hline Age, years: ${ }^{a}$ mean (s.d.) & $21.64(3.23)$ & $22.49(3.88)$ & 0.40 \\
\hline NART IQ, ${ }^{\text {b }}$ mean (s.d.) & $97.28(11.28)$ & $101.91(8.41)$ & 0.13 \\
\hline Illness duration, years: median (minimum-maximum) & $0.06(0.01-0.20)$ & & \\
\hline \multicolumn{4}{|l|}{ PANSS score, ${ }^{\mathrm{c}}$ mean (s.d.) } \\
\hline Positive & $21.5(8.14)$ & & \\
\hline Negative & $14.86(6.08)$ & & \\
\hline Disorganisation & $28.7(8.98)$ & & \\
\hline Excitement & $23.75(6.35)$ & & \\
\hline Emotional distress & $22.6(4.82)$ & & \\
\hline \multicolumn{4}{|c|}{$\begin{array}{l}\text { NART, National Adult Reading Test; PANSS Positive and Negative Syndrome Scale. } \\
\text { a. Age was compared using Student's } t \text {-tests. } \\
\text { b. NART IQ data were unavailable for six patients and three controls. NART IQ was compared using the Mann-Whitney U-test. } \\
\text { c. PANSS factor scores based on van der Gaag et } a l^{53} \text { Data unavailable for six patients. }\end{array}$} \\
\hline
\end{tabular}


four-category classification system that described the incidence of the paracingulate sulcus in both hemispheres for each individual. The four categories were: present in the left and absent in the right hemisphere $(n=9)$; present in both hemispheres $(n=6)$; absent in both hemispheres $(n=6)$; or absent in the left and present in the right hemisphere $(n=5)$. Each patient was classified according to this system and controls were then randomly selected from a larger database and individually matched to each patient on the basis of this classification, gender and age. Since paracingulate sulcus and superior rostral sulcus classifications tend to be related, this procedure also resulted in good matching for superior rostral sulcus morphology. In the right hemisphere, 8 patients and 8 controls were classified as having a continuous superior rostral sulcus, and the remaining 18 patients and 18 controls were classified as having a separate superior rostral sulcus. In the left hemisphere, 7 patients and 12 controls had a continuous classification, whereas 19 patients and 14 controls were classified as showing a separate pattern. Although there can still be considerable variation in the rostro-caudal extent of the paracingulate sulcus within participants classified as 'present', we have previously shown that such variations do not have a major influence on the measures examined in this study. ${ }^{17}$

\section{Cortical surface reconstruction}

The white (i.e. grey/white matter boundary) and pial (grey/ cerebrospinal fluid boundary) surfaces of the cortical ribbon were tessellated with a triangular mesh comprising $~ 150000$ vertices per hemisphere using methods described in detail elsewhere $e^{27-29}$ and as implemented in the Freesurfer software package (http:// surfer.nmr.mgh.harvard.edu). The surfaces were reconstructed using the raw, unaligned images in native space to avoid unnecessary interpolation and resampling, and were visually inspected and corrected for accuracy as per guidelines on the Freesurfer website. Reconstructing these surfaces enabled calculation of surface area, grey matter volume and mean cortical thickness for each of six anterior cingulate cortex regions per hemisphere. Distinguishing between these three measures is important given that volume, the most widely used metric in anatomical MRI studies in patients with bipolar disorder, is a relatively diffuse measure that reflects the product of a cortical region's surface area and thickness. Consequently, changes in one or the other parameter can be obscured unless both are assessed independently an important consideration when attempting to characterise relatively subtle changes early in the illness course.

Each of the measures were calculated according to previously described methods. ${ }^{28}$ Briefly, cortical thickness was calculated at each surface point by finding the vertex on the pial surface closest to a given point on the white surface (and vice versa) and averaging these two values. As the surfaces are generated with sub-voxel resolution, the resulting thickness measures are estimated with sub-millimeter precision and have been validated against post-mortem specimens. ${ }^{30}$ The thickness values for each surface point in the regions of interest were averaged to obtain the mean thickness for that region. Regional surface area was calculated by summing the area of the triangle faces included in each region of interest for the white and pial surfaces separately, and then taking the average of the two. Grey matter volumes were calculated as the product of surface area and thickness at each surface point, averaged across all pial and white matter vertices in each region of interest.

\section{Region-of-interest delineation}

The parcellation protocol divides the $\mathrm{ACC}_{\mathrm{L}}$ and $\mathrm{ACC}_{\mathrm{P}}$ into dorsal, rostral and subcallosal regions, yielding six regions of interest per hemisphere. Boundaries separating the dorsal, rostral and subcallosal regions were designed to approximate previously identified functional subdivisions within the area. ${ }^{1,2}$ Boundaries distinguishing between the $\mathrm{ACC}_{\mathrm{L}}$ and $\mathrm{ACC}_{\mathrm{P}}$ varied in accordance with paracingulate sulcus and superior rostral sulcus variability and were based on post-mortem work documenting how cytoarchitectonic areas in the region vary in accordance with sulcal morphology ${ }^{26}$ (online Fig. DS1). A more detailed justification and description of these boundaries has been provided elsewhere. ${ }^{19}$ We have recently shown that reliabilities for this method are satisfactory (all $>0.8$, with most $>0.9) .{ }^{17}$

\section{Intracranial volume}

Intracranial volume was calculated for each individual to control for any group differences in brain size using a previously described, reliable method. ${ }^{23}$

\section{Statistical analyses}

All analyses were performed using SPSS 12.0 for Windows. Regional grey matter volumes, cortical thickness and surface area were analysed with mixed within-participant and betweenparticipants ANOVA, with hemisphere (left or right), region (dorsal, rostral and subcallosal) and cortex $\left(\mathrm{ACC}_{\mathrm{L}}\right.$ or $\left.\mathrm{ACC}_{\mathrm{P}}\right)$ as within-participant factors, and diagnosis and gender as betweenparticipants factors. Gender was included as a factor in the analyses to determine whether diagnostic differences varied for males and females. There is growing evidence that gender interacts with diagnosis in affecting brain morphology, including the anterior cingulate cortex, in other psychotic disorders, ${ }^{31,32}$ but such effects have seldom been investigated in bipolar disorder. Volume, area and thickness were treated as independent measures analysed using separate models to simplify interpretation of the findings. This approach allows inferences regarding group differences within each measure, but does not allow any conclusions regarding relative differences across measures (see Limitations for further discussion). As sphericity assumptions were invariably violated, main effects and interactions were evaluated using Greenhouse-Geisser corrected degrees of freedom, with $\alpha=0.05$. Post hoc contrasts were evaluated against a Bonferroni-adjusted $\alpha$ to correct for multiple comparisons. Effect sizes, expressed as Cohen's $d$, are also reported for these contrasts (negative values indicate a decrease in the patient group). Only effects involving diagnosis are reported, as these were the primary focus of the current study.

We corrected grey matter volume and surface area estimates for intracranial volume using equations described by Free et al. ${ }^{33}$ For cortical thickness, we report results of analyses of uncorrected values since the anatomical significance of the relationship between thickness and intracranial volume is unclear. $^{34}$ (We note that separate analyses that did covary for intracranial volume yielded similar findings.)

\section{Results}

\section{Anterior cingulate cortex morphometry}

Group means for grey matter volume, surface area and cortical thickness are presented in Table 2. For grey matter volume, there was no main effect of diagnosis $(F(1,48)=0.887, P=0.351)$ or diagnosis $\times$ gender interaction $(F(1,48)=0.164, P=0.687)$, nor did diagnosis interact with any of the within-participant factors. Similarly, there was no significant main effect of diagnosis $(F(1,48)=1.248, \quad P=0.270), \quad$ diagnosis $\times$ gender interaction 


\begin{tabular}{|c|c|c|c|c|c|c|c|c|c|c|c|c|}
\hline & \multicolumn{2}{|c|}{ Dorsal $\mathrm{ACC}_{\mathrm{L}}$} & \multicolumn{2}{|c|}{ Dorsal $A_{C C}$} & \multicolumn{2}{|c|}{ Rostral $\mathrm{ACC}_{\mathrm{L}}$} & \multicolumn{2}{|c|}{ Rostral $\mathrm{ACC}_{\mathrm{P}}$} & \multicolumn{2}{|c|}{ Subcallosal $A C C_{L}$} & \multicolumn{2}{|c|}{ Subcallosal $\mathrm{ACC}_{\mathrm{P}}$} \\
\hline & Left & Right & Left & Right & Left & Right & Left & Right & Left & Right & Left & Right \\
\hline \multicolumn{13}{|c|}{ Patients with bipolar disorder } \\
\hline Volume, $\mathrm{mm}^{3}$ : mean & 1916.31 & 2292.52 & 1495.45 & 1366.34 & 1523.72 & 1985.13 & 2517.24 & 2554.05 & 285.40 & 281.71 & 279.48 & 225.68 \\
\hline (s.d.) & $\begin{array}{l}(472.77) \\
675.92\end{array}$ & $(607.52)$ & (697.03) & (599.53) & (873.83) & (642.88) & (1067.52) & (701.41) & (141.37) & (123.42) & (157.65) & (130.85) \\
\hline $\begin{array}{l}\text { Area, } \mathrm{mm}^{2} \text { : mean } \\
\text { (s.d.) }\end{array}$ & $\begin{array}{c}675.93 \\
(132.10)\end{array}$ & $\begin{array}{c}806.13 \\
(186.52)\end{array}$ & $\begin{array}{l}517.90 \\
(240.53)\end{array}$ & $\begin{array}{c}467.32 \\
(196.53)\end{array}$ & $\begin{array}{c}478.71 \\
(286.64)\end{array}$ & $\begin{array}{c}633.88 \\
(204.46)\end{array}$ & $\begin{array}{l}894.04 \\
(362.95)\end{array}$ & $\begin{array}{l}873.15 \\
(247.03)\end{array}$ & $\begin{array}{l}105.02 \\
(48.97)\end{array}$ & $\begin{array}{l}86.74 \\
(40.79)\end{array}$ & $\begin{array}{c}89.99 \\
(52.99)\end{array}$ & $\begin{array}{l}74.13 \\
(50.54)\end{array}$ \\
\hline $\begin{array}{l}\text { Thickness, mm: mean } \\
\text { (s.d.) }\end{array}$ & $\begin{array}{l}2.79 \\
(0.29)\end{array}$ & $\begin{array}{l}2.79 \\
(0.26)\end{array}$ & $\begin{array}{l}2.84 \\
(0.24)\end{array}$ & $\begin{array}{l}2.86 \\
(0.22)\end{array}$ & $\begin{array}{l}3.14 \\
(0.21)\end{array}$ & $\begin{array}{l}3.11 \\
(0.30)\end{array}$ & $\begin{array}{l}2.75 \\
(0.23)\end{array}$ & $\begin{array}{l}2.89 \\
(0.21)\end{array}$ & $\begin{array}{l}2.70 \\
(0.34)\end{array}$ & $\begin{array}{c}3.25 \\
(0.39)\end{array}$ & $\begin{array}{l}3.18 \\
(0.54)\end{array}$ & $\begin{array}{l}3.29 \\
(0.60)\end{array}$ \\
\hline \multicolumn{13}{|l|}{ Controls } \\
\hline $\begin{array}{l}\text { Volume, } \mathrm{mm}^{3} \text { : mean } \\
\text { (s.d.) }\end{array}$ & $\begin{array}{r}2014.99 \\
(618.19)\end{array}$ & $\begin{array}{l}2273.84 \\
(508.31)\end{array}$ & $\begin{array}{l}1431.36 \\
(754.26)\end{array}$ & $\begin{array}{l}1338.16 \\
(484.55)\end{array}$ & $\begin{array}{l}1786.26 \\
(991.33)\end{array}$ & $\begin{array}{l}1835.21 \\
(891.62)\end{array}$ & $\begin{array}{l}2121.12 \\
(890.84)\end{array}$ & $\begin{array}{r}2382.65 \\
(676.72)\end{array}$ & $\begin{array}{c}313.15 \\
(192.71)\end{array}$ & $\begin{array}{c}250.85 \\
(132.98)\end{array}$ & $\begin{array}{c}216.86 \\
(157.22)\end{array}$ & $\begin{array}{r}215.51 \\
(124.98)\end{array}$ \\
\hline Area, $\mathrm{mm}^{2}:$ mean & 718.55 & 797.68 & 499.84 & 467.12 & 550.66 & 582.09 & 729.44 & 816.94 & 110.69 & 82.38 & 73.91 & 68.73 \\
\hline (s.d.) & (180.88) & $(167.37)$ & (241.03) & $(155.37)$ & (305.78) & $(275.63)$ & $(288.46)$ & $(215.12)$ & (61.58) & (41.31) & $(55.89)$ & (47.83) \\
\hline Thickness, mm: mean & 2.76 & 2.85 & 2.80 & 2.82 & 3.18 & 3.07 & 2.81 & 2.87 & 2.70 & 2.97 & 3.06 & 3.12 \\
\hline (s.d.) & $(0.28)$ & $(0.18)$ & $(0.19)$ & $(0.19)$ & $(0.38)$ & $(0.21)$ & $(0.22)$ & $(0.20)$ & $(0.45)$ & $(0.46)$ & $(0.42)$ & $(0.43)$ \\
\hline
\end{tabular}

$(F(1,48)=0.263, P=0.610)$ or interaction between diagnosis and any within-participant factor for surface area.

For cortical thickness, there was no main effect of diagnosis $(F(1,48)=0.705, \quad P=0.405) \quad$ or $\quad$ diagnosis $\times$ gender interaction $(F(1,48)=1.001, P=0.322)$, although there was a significant diagnosis $\times$ hemisphere $\times$ gender interaction $(F(1,48)=5.790$, $P=0.020$ ). As can be seen in Fig. 1 , this effect was largely driven by male patients showing increased thickness in the right subcallosal areas. Post hoc contrasts revealed that male patients showed a significant thickness increase in the right subcallosal $\mathrm{ACC}_{\mathrm{L}}$, with no other differences reaching statistical significance (Table 3).

\section{Medication effects}

Excluding the seven patients taking lithium did not change the cortical thickness finding (hemisphere $\times$ gender $\times$ diagnosis interaction: $F(1,34)=8.796, P=0.005)$. To investigate whether antipsychotic class had any effect, patients taking typicals were matched to those taking atypicals for paracingulate sulcus morphology using the procedures described above. Any patients who could not be matched were excluded from the analysis, resulting in eight patients taking typicals being compared with eight taking atypicals. This analysis revealed no significant main effect of antipsychotic class on cortical thickness $(F(1,14)=0.131, \quad P=0.723)$ and no interactions between medication class and any of the within-participant factors.

\section{Discussion}

This study implemented a novel surface-based approach to characterise anatomical abnormalities of the anterior cingulate cortex in patients with bipolar disorder experiencing their first episode of psychosis. Relative to controls matched for age, gender and paracingulate sulcus, male patients showed a significant thickness increase in the right subcallosal $\mathrm{ACC}_{\mathrm{L}}$. No significant differences were identified for grey matter volume or surface area, or for any measure in the female group. The fact that only thickness, but not surface area or volume, was different highlights the importance of distinguishing between these measures when assessing anatomical changes in psychiatric disorders and suggests thickness may be a particularly sensitive metric for detecting such changes, especially in early illness stages. Importantly, this is the first study to match patients and controls for sulcal variability, indicating these differences are unlikely to be caused by group biases in cortical folding patterns.

\section{Past findings}

Differences in subcallosal areas were expected, although the direction of the difference (i.e. grey matter increase in patients) was not. Several authors have emphasised a primary role for subcallosal grey matter reductions, particularly in the left hemisphere, in the pathophysiology of bipolar disorder, $3,12,20$ but past MRI studies of the anterior cingulate cortex in bipolar disorder have reported variable findings with respect to the laterality, subregion and nature (grey matter increase or decrease) of the abnormality. ${ }^{4-8,12}$ The majority of these studies have been conducted in patients with prolonged illness, although four other studies of patients with first-episode affective psychosis have also yielded contradictory findings. ${ }^{1-13}$ Direct comparison between these reports and our findings is complicated by differences in the methods used and the fact that we had insufficient power to appropriately examine the effects of family history. This is an important consideration, given that two independent studies have previously found left subcallosal reductions in familial patients. ${ }^{4,12}$ By way of speculation, the fact that the majority of our sample did not report a family history of affective illness suggests that left-sided abnormalities may be more prominent in familial cases, whereas right-sided changes may characterise sporadic patients.

Prior neglect of the potential effect of paracingulate sulcus variability on the findings is also likely to contribute to inconsistent findings in the literature. We have previously shown that paracingulate sulcus variability can have a large and complex effect on regional volume, surface area and thickness in the anterior cingulate cortex ${ }^{17,19}$ and that, as a group, patients with bipolar disorder are less likely to display this sulcus than healthy controls. ${ }^{15}$ This means that spurious group differences in estimates of regional grey matter such as volume and thickness can arise if patients and controls are not well matched for this anatomical variation. Consequently, inconsistent and contradictory findings of anterior cingulate cortex changes in patients with bipolar disorder may partly reflect differences in regional cortical folding patterns rather than true changes in the relative amount of grey matter in the region.

When such sulcal variability is accounted for, our findings suggest that first-episode psychosis in bipolar disorder is associated with a thickness increase in the right subcallosal ACC $_{\mathrm{L}}$ of male patients. Relatively few studies of bipolar disorder have examined gender effects on anterior cingulate cortex morphometry in detail. Drevets et $a l^{4}$ found a left subcallosal anterior 

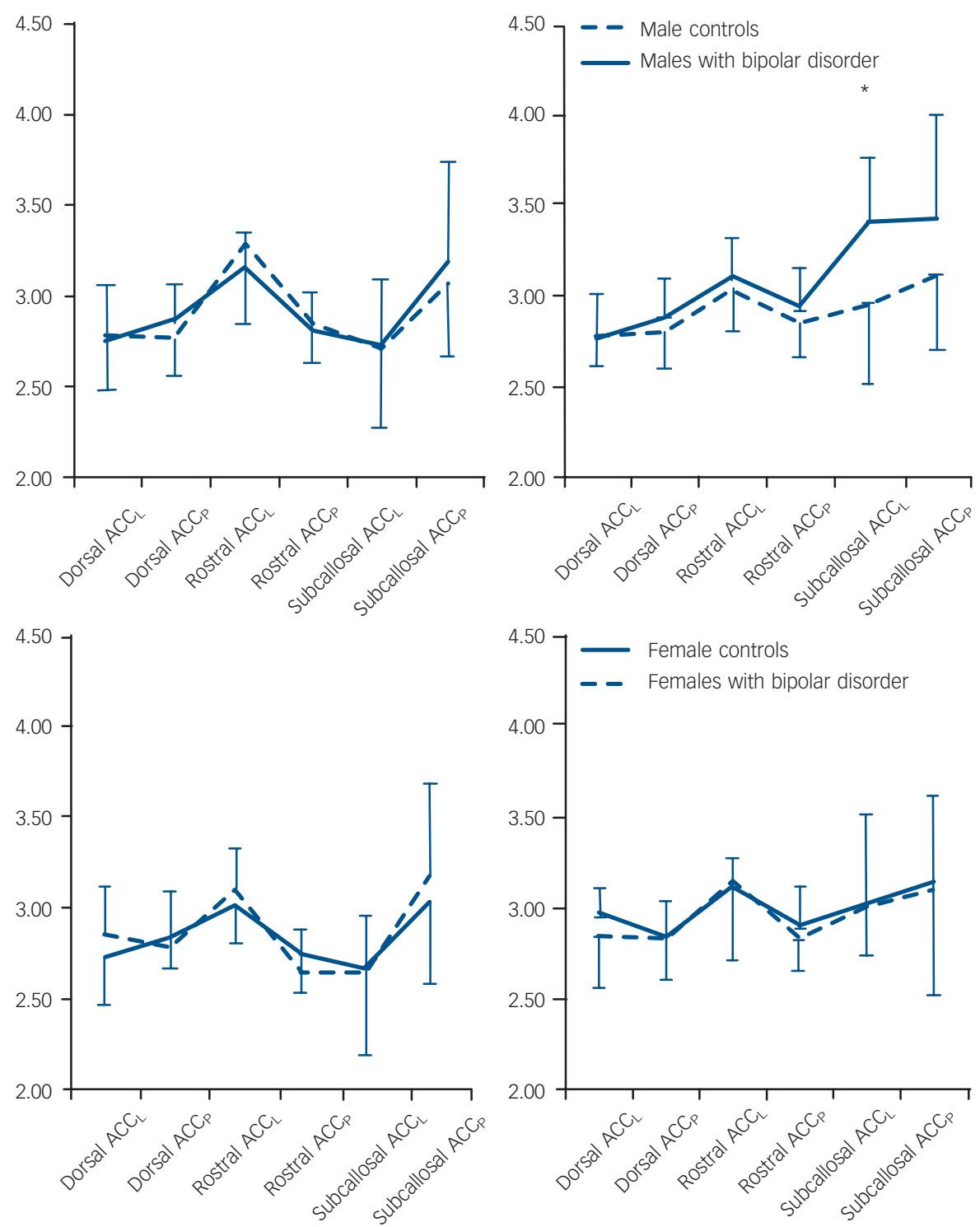

Fig. 1 Mean thickness for male and female patients and controls in each limbic $\left(A C C_{L}\right)$ and paralimbic $\left(A C C_{P}\right)$ anterior cingulate cortex subregion.

Error bars represent standard deviations. Left column presents data for left hemisphere regions; right column presents data for right hemisphere regions. ${ }^{*} P<0.05$, corrected.

cingulate cortex reduction while statistically covarying for gender in patients with chronic bipolar disorder, ${ }^{4}$ whereas another study of a similar region in a predominantly female sample found no group differences, ${ }^{35}$ suggesting the differences are more pronounced in males. Kaur et $a l^{36}$ failed to identify a gender $\times$ diagnosis interaction in their study of the entire anterior cingulate gyrus, although they studied a paediatric sample.

To date, only three other studies have found grey matter increases in the anterior cingulate cortex of patients with bipolar disorder. Adler $e t a l^{37}$ reported an increase in a region corresponding to our rostral $\mathrm{ACC}_{\mathrm{L}} / \mathrm{ACC}_{\mathrm{P}}$ in a sample of patients with long-term illness. More recently, Bearden et al ${ }^{10}$ found evidence of increased grey matter in the anterior cingulate cortex of patients with established bipolar disorder, although they attributed their findings to the effects of lithium, since a subsample of patients not taking lithium at the time of scanning showed no evidence of such changes, and a significant, inverted U-shaped relationship was observed between anterior cingulate cortex grey matter and duration of exposure to lithium. However, another recent study of patients with bipolar disorder experiencing their first episode of mania ${ }^{11}$ also reported increased anterior cingulate cortex grey matter in a sample where only 1 patient (out of 33) was being treated with lithium. When coupled with our finding that excluding patients taking lithium did not change the results, this suggests not all grey matter increases can be attributed to the effects of this drug. It should be noted, however, that the grey matter increase reported by Adler et al ${ }^{11}$ was in the caudal, rather than subcallosal anterior cingulate cortex. Reasons for this discrepancy include that Adler et al did not consider the influence of paracingulate sulcus variability on their findings and that they did not examine gender $\times$ diagnosis interactions. Furthermore, our sample was composed entirely of patients with bipolar disorder experiencing psychotic symptoms, whereas Adler et al ${ }^{11}$ did not report how many of their patients were experiencing such symptoms. This may be an important distinction, given emerging evidence for a distinct phenotype in this subgroup of patients. ${ }^{38}$ Further investigation of the neuroanatomical changes associated with psychotic and non-psychotic bipolar disorder will therefore be an important avenue of future research.

The gender-specific nature of our findings, coupled with the direction and laterality of the thickness difference (i.e. we found a right-sided increase, rather than decrease, in our patient group), 


\begin{tabular}{|c|c|c|c|c|c|c|}
\hline & \multicolumn{3}{|c|}{ Males } & \multicolumn{3}{|c|}{ Females } \\
\hline & $F$ & $d$ & $P$ & $F$ & $d$ & $P$ \\
\hline \multicolumn{7}{|l|}{ Left } \\
\hline \multicolumn{7}{|l|}{ Dorsal } \\
\hline $\mathrm{ACC}_{\mathrm{L}}$ & 0.097 & -0.108 & 0.757 & 0.837 & 0.476 & 0.365 \\
\hline $\mathrm{ACC}_{\mathrm{P}}$ & 1.820 & 0.536 & 0.184 & 0.317 & -0.236 & 0.576 \\
\hline \multicolumn{7}{|l|}{ Rostral } \\
\hline $\mathrm{ACC}_{\mathrm{L}}$ & 1.456 & -0.389 & 0.233 & 0.449 & 0.436 & 0.506 \\
\hline $\mathrm{ACC}_{\mathrm{P}}$ & 0.127 & -0.138 & 0.723 & 1.336 & -0.503 & 0.254 \\
\hline \multicolumn{7}{|c|}{ Subcallosal } \\
\hline $\mathrm{ACC}_{\mathrm{L}}$ & 0.030 & 0.063 & 0.863 & 0.014 & -0.055 & 0.907 \\
\hline $\mathrm{ACC}_{\mathrm{P}}$ & 0.442 & 0.241 & 0.509 & 0.375 & 0.293 & 0.543 \\
\hline \multicolumn{7}{|l|}{ Right } \\
\hline \multicolumn{7}{|l|}{ Dorsal } \\
\hline $\mathrm{ACC}_{\mathrm{L}}$ & 0.024 & -0.058 & 0.878 & 1.640 & -0.578 & 0.206 \\
\hline $\mathrm{ACC}_{\mathrm{P}}$ & 1.162 & 0.397 & 0.286 & 0.013 & -0.053 & 0.909 \\
\hline \multicolumn{7}{|l|}{ Rostral } \\
\hline $\mathrm{ACC}_{\mathrm{L}}$ & 0.442 & 0.283 & 0.509 & 0.026 & 0.063 & 0.873 \\
\hline $\mathrm{ACC}_{\mathrm{P}}$ & 1.402 & 0.425 & 0.242 & 0.621 & -0.383 & 0.434 \\
\hline \multicolumn{7}{|c|}{ Subcallosal } \\
\hline $\mathrm{ACC}_{\mathrm{L}}$ & 9.994 & 1.146 & $0.003^{*}$ & 0.004 & -0.031 & 0.949 \\
\hline $\mathrm{ACC}_{\mathrm{P}}$ & 2.756 & 0.616 & 0.103 & 0.038 & -0.089 & 0.847 \\
\hline
\end{tabular}

is interesting in light of recent evidence regarding the role of the subcallosal anterior cingulate cortex in regulating the body's physiological stress response, particularly as mediated through the hypothalamic-pituitary-adrenal (HPA) axis. The subcallosal anterior cingulate cortex is heavily connected with the hypothalamus, amygdala and brainstem autonomic nuclei. ${ }^{39}$ Rodent work has shown that lesions to the right, but not left, ventral medial prefrontal cortex (which includes the subcallosal anterior cingulate cortex) significantly decrease corticosterone levels in response to external stressors, ${ }^{40}$ suggesting that the right subcallosal anterior cingulate cortex plays an important role in activating the HPA axis. It is, therefore, plausible that although lesions to this area reduce HPA activation, increased thickness may reflect a hyperfunctional abnormality that produces an excessive stress response. Heightened HPA activity has been demonstrated in patients with first-episode psychosis, ${ }^{41}$ and although the precise mechanism through which a thickness increase might lead to this excessive stress response remains unclear, a link between the two is suggested by evidence that other brain regions implicated in HPA axis activation, such as the amygdala and pituitary gland, are also enlarged in patients with first-episode affective psychosis. ${ }^{21,42}$ In this context, two notable findings are that: (a) patients with bipolar disorder show increased anatomical connectivity between the subcallosal anterior cingulate cortex and amygdala; ${ }^{43}$ and (b) volume of the pituitary gland, which is correlated with peripheral measures of HPA activation, ${ }^{44}$ is increased in ultra-high-risk individuals who eventually develop an affective psychosis, with the enlargements being most pronounced in individuals scanned in closer temporal proximity to psychosis onset. ${ }^{45}$ It is unclear whether an increase in anterior cingulate cortex thickness such as that observed in our data would also precede psychosis onset, although these findings do support the notion that size increases in brain regions implicated in HPA regulation are associated with an elevated stress response. Chronic hyperactivity of this circuit may account for why reports of volumetric reductions in the anterior cingulate cortex are more common in samples with chronic illness, ${ }^{4,5,35}$ given that prolonged elevation of corticosterone levels has been associated with decreased volume in both rodents and humans. ${ }^{46,47}$
Taken together, these findings suggest that relative hypertrophy in brain regions critical for regulating HPA axis activation (i.e. the anterior cingulate cortex, amygdala and pituitary) are associated with an elevated stress response around the time of psychosis onset that ultimately causes volumetric contractions in later illness stages. In this context, the gender-specific nature of our findings may be related to gender differences in HPA reactivity, which is known to be elevated in males relative to females. ${ }^{48}$ It should be noted, however, that left-sided reductions have been observed in patients with familial bipolar disorder, ${ }^{12}$ with Drevets et $a l^{20}$ positing that such changes may disinhibit right-sided activation of physiological stress responses in familial patients. Thus, although the effects of paracingulate sulcus variability on these earlier findings are unknown and the precise effects of family history remain to be characterised, it is possible that lateralised pathology of the subcallosal anterior cingulate cortex differs in sporadic and familial cases. The net effect of both would be to increase HPA reactivity, albeit through distinct mechanisms. Future work examining the role of stress, gender and family illness history in influencing the longitudinal course of neuroanatomical abnormalities in bipolar disorder will be critical for characterising these relationships further.

\section{Limitations}

In this study, we defined 'first-episode' as first contact with psychiatric services for a psychotic episode and we did not control for the effects of prodromal symptoms, duration of untreated psychosis or pre-psychotic affective episodes. The effects of such characteristics can only be unambiguously determined through prospective work, although one recent voxel-based study found no effect of duration of untreated psychosis (retrospectively assessed) on anterior cingulate cortex grey matter, ${ }^{49}$ suggesting that any such influences on our region-of-interest measures may be minimal. We also note that there was no significant correlation between thickness in this region and duration of psychosis in our patient sample (Spearman's rho $=-0.121, P=0.574$ ).

Excluding patients taking lithium did not significantly alter our findings, suggesting they do not reflect grey matter increases known to be related to mood stabiliser treatment. ${ }^{10}$ Similarly, 
we found no evidence for an effect of antipsychotic class, although our power for detecting such an effect was limited. Past studies of such effects have yielded inconsistent findings, with one voxelbased investigation reporting that, relative to drug-free patients with psychosis (comprising both schizophrenia and affective psychosis), those taking typical antipsychotics had less anterior cingulate cortex grey matter, whereas those taking atypicals showed no significant changes in this region. ${ }^{50}$ In contrast, recent work using regionof-interest techniques has suggested that anterior cingulate cortex volume is positively correlated with exposure to typical antipsychotics and negatively correlated with exposure to atypicals. ${ }^{51}$ However, this study also failed to find a difference in the rate of volumetric change between patients with schizophrenia taking typicals or atypicals in the first 2-3 years following psychosis onset, suggesting that the effects of differential antipsychotic exposure on anterior cingulate cortex morphometry are minimised in the first few years of illness. Although we cannot determine whether similar changes to those reported in this paper would be observed in a medication-naïve sample, the fact that the median illness duration in our sample was $\sim 3$ weeks (the longest was 10 weeks) suggests that any effects of antipsychotic medication were minimal.

We deemed our retrospective paracingulate sulcus matching procedure to be an efficient means for matching patients and controls on this important parameter since prospective matching would require very large samples (i.e. its morphology is only ascertainable after scanning). It should be noted that controls, but not patients, were selected to meet the matching criteria, meaning that no biases were introduced into the patient sample, as this group represented all patients for whom data were available. Although the matching procedure may have introduced some sampling bias in the control group, this bias would have minimised any differences between patients and controls, since the controls were being chosen explicitly because their brain had a similar morphology to that of the patient group. (Preliminary analyses indicated no significant differences between controls selected and not selected with respect to National Adult Reading Test ${ }^{52}$ IQ.) Matching patients and controls for both paracingulate sulcus and superior rostral sulcus morphology would require very large samples and was not possible in the current study. However, since these two morphological variations tend to be related, our paracingulate sulcus matching also resulted in good matching for superior rostral sulcus morphology. Importantly, superior rostral sulcus morphology was perfectly matched in the right hemisphere, where the primary thickness differences were, suggesting superior rostral sulcus differences cannot account for our findings.

Our attempt to account for the functional and anatomical diversity of the anterior cingulate cortex resulted in a highly multivariate data-set with many comparisons being made. Our analyses therefore attempted to strike an appropriate balance between control of type I and type II errors, while minimising complexity. To simplify analysis and interpretation of the findings, we treated grey matter volume, area and thickness as independent measures that were analysed using separate models. As we treated these measures independently, we evaluated main effects and interactions in these models using uncorrected significance thresholds, although the post hoc contrasts used to characterise the main finding of this work were tested using very stringent criteria (i.e. Bonferroni correction for 12 comparisons). This, coupled with the large effect size associated with the subcallosal difference (Cohen's $d>1$ ), indicates the effect is robust and unlikely to be a false positive. However, our failure to find differences in other regions may have reflected a lack of power. Table 3 indicates there were several regions in which moderate effect sizes were obtained (including in female patients), suggesting they warrant further investigation in larger samples. Such work should also investigate the relationship and interactions between changes in volume, area and cortical thickness, rather than treating them independently.

\section{Future study}

Our novel surface-based approach enabled us to derive a detailed characterisation of anterior cingulate cortex abnormalities in the early stages of bipolar psychosis. By explicitly accounting for individual differences in sulcal morphology, we ensured that our results are not an artifact of group differences in local cortical folding patterns. Our findings provide an impetus for future research examining the longitudinal course of neuroanatomical abnormalities in bipolar disorder, while also considering the influences of family illness history and gender differences in stress responses.

\begin{abstract}
Alex Fornito, PhD, Melbourne Neuropsychiatry Centre, Department of Psychiatry, and Department of Psychology, School of Behavioural Science, Faculty of Medicine, Dentistry and Health Sciences, University of Melbourne, Australia; Murat Yücel, PhD, Melbourne Neuropsychiatry Centre, Department of Psychiatry, and ORYGEN Research Centre, University of Melbourne, Australia; Stephen J. Wood, PhD, Melbourne Centre, University of Melbourne, Australia; Stephen J. Wood, PhD, Melbourne
Neuropsychiatry Centre, Department of Psychiatry, University of Melbourne, Australia; Andreas Bechdolf, MD, MSC, ORYGEN Research Centre, Department of Psychiatry, University of Melbourne, Australia, and Department of Psychiatry and Psychotherapy, University of Cologne, Germany: Simon Carter, BSC, ORYGEN Research Centre, Department of Psychiatry, University of Melbourne, Australia; Chris Adamson, BSC (Hons), Department of Electrical \& Electronic Engineering and Howard Florey Institute, University of Melbourne, Australia; Dennis Velakoulis, MBBS, FRANCP, Melbourne University of Melbourne, Australia; Dennis Velakoulis, MBBS, FRANCP, Melbourne
Neuropsychiatry Centre, Department of Psychiatry, University of Melbourne, Australia; Michael M. Saling, PhD, Department of Psychology, School of Behavioural Science, Faculty of Medicine, Dentistry and Health Sciences, University of Melbourne, Australia; Patrick D. McGorry, MBBS, FRANZCP, PhD, ORYGEN Research Centre, Department of Psychiatry, University of Melbourne, Australia; Christos Pantelis, MBBS, FRANCP, MRCPsych, MD, Melbourne Neuropsychiatry Centre, Department of Psychiatry, and Howard Florey Institute University of Melbourne, Australia
\end{abstract}

Correspondence: Dr Alex Fornito, Melbourne Neuropsychiatry Centre, Levels 2 and 3, National Neuroscience Facility, 161 Barry Street, Carlton South, VIC 3053, Australia.Email: fornitoa@unimelb.edu.au

First received 22 Dec 2008, final revision 21 Aug 2008, accepted 29 Oct 2008

\section{Funding}

This research was supported by the Melbourne Neuropsychiatry Centre (Sunshine Hospital), Department of Psychiatry, The University of Melbourne; the National Health and Medical Research Council (NHMRC) (ID 236175; 350241); and the lan Potter Foundation. Neuroimaging analysis was facilitated by the Neuropsychiatry Imaging Laboratory managed by Ms Bridget Soulsby at the Melbourne Neuropsychiatry Centre and supported by Neurosciences Victoria. S.J.W. was supported by a NHMRC Clinical Career Development Award and a NARSAD Young Investigator Award. A.F. was supported by a JN Peters Fellowship and a NHMRC CJ Martin Fellowship (ID 454797).

\section{Acknowledgement}

The authors thank Dr Sue M. Cotton for helpful comments regarding this manuscript.

\section{References}

1 Devinsky O, Morrell MJ, Vogt BA. Contributions of anterior cingulate cortex to behaviour. Brain 1995; 118: 279-306.

2 Bush G, Luu P, Posner MI. Cognitive and emotional influences in anterior cingulate cortex. Trends Cogn Sci 2000; 4: 215-22.

3 Harrison PJ. The neuropathology of primary mood disorder. Brain 2002; 125 (Pt 7): 1428-49.

4 Drevets WC, Price JL, Simpson JR Jr, Todd RD, Reich T, Vannier M, et al. Subgenual prefrontal cortex abnormalities in mood disorders. Nature 1997; 386: 824-7.

5 Sharma V, Menon R, Carr TJ, Densmore M, Mazmanian D, Williamson PC. An MRI study of subgenual prefrontal cortex in patients with familial and nonfamilial bipolar I disorder. J Affect Disord 2003; 77: 167-71.

6 Brambilla P, Nicoletti MA, Harenski K, Sassi RB, Mallinger AG, Frank E, et al. Anatomical MRI study of subgenual prefrontal cortex in bipolar and unipolar subjects. Neuropsychopharmacology 2002; 27: 792-9.

7 Zimmerman ME, DelBello MP, Getz GE, Shear PK, Strakowski SM. Anterior cingulate subregion volumes and executive function in bipolar disorder. Bipolar Disord 2006; 8: 281-8. 
8 Lyoo IK, Kim MJ, Stoll AL, Demopulos CM, Parow AM, Dager SR, et al. Frontal lobe gray matter density decreases in bipolar I disorder. Biol Psychiatry 2004; 55: 648-51.

9 Fornito A, Malhi GS, Lagopoulos J, Ivanovski B, Wood SJ, Saling MM, et al. Anatomical abnormalities of the anterior cingulate and paracingulate cortex in patients with bipolar I disorder. Psychiatry Res 2008; 162: 123-32.

10 Bearden CE, Thompson PM, Dalwani M, Hayashi KM, Lee AD, Nicoletti M, et al. Greater cortical gray matter density in lithium-treated patients with bipolar disorder. Biol Psychiatry 2007; 62: 7-16.

11 Adler CM, DelBello MP, Jarvis K, Levine A, Adams J, Strakowski SM. Voxelbased study of structural changes in first-episode patients with bipolar disorder. Biol Psychiatry 2007; 61: 776-81.

12 Hirayasu Y, Shenton ME, Salisbury DF, Kwon JS, wible CG, Fischer IA, et al. Subgenual cingulate cortex volume in first-episode psychosis. Am J Psychiatry 1999; 156: 1091-3.

13 Kubicki M, Shenton ME, Salisbury DF, Hirayasu Y, Kasai K, Kikinis R, et al. Voxel-based morphometric analysis of gray matter in first episode schizophrenia. Neuroimage 2002; 17: 1711-9.

14 Farrow TF, Whitford TJ, Williams LM, Gomes L, Harris AW. Diagnosis-related regional gray matter loss over two years in first episode schizophrenia and bipolar disorder. Biol Psychiatry 2005; 58: 713-23.

15 Fornito A, Malhi GS, Lagopoulos J, Ivanovski B, Wood SJ, Velakoulis D, et al. In vivo evidence for early neurodevelopmental anomaly of the anterior cingulate cortex in bipolar disorder. Acta Psychiatr Scand 2007; 116: 467-72.

16 Paus T, Otkay N, Caramanos Z, MacDonald D, Zijdenbos A, D'Avirro D, et al. In vivo morphometry of the intrasulcal gray matter in the human cingulate, paracingulate, and superior rostral sulci. Hemispheric asymmetries, gender differences and probability maps. J Comp Neurol 1996; 376: 664-73.

17 Fornito A, Wood SJ, Whittle S, Fuller J, Adamson C, Saling MM, et al. Variability of the paracingulate sulcus and morphometry of the medial frontal cortex. Associations with cortical thickness, surface area, volume, and sulcal depth. Hum Brain Mapp 2008; 29: 222-36.

18 Fornito A, Yucel M, Wood SJ, Proffitt T, McGorry PD, Velakoulis D, et al. Morphology of the paracingulate sulcus and executive cognition in schizophrenia. Schizophr Res 2006; 88: 192-7.

19 Fornito A, Whittle S, Wood SJ, Velakoulis D, Pantelis C, Yucel M. The influence of sulcal variability on morphometry of the human anterior cingulate and paracingulate cortex. Neuroimage 2006; 33: 843-54.

20 Drevets WC, Ongur D, Price JL. Neuroimaging abnormalities in the subgenual prefrontal cortex: implications for the pathophysiology of familial mood disorders. Mol Psychiatry 1998; 3: 220-6.

21 American Psychiatric Association. Diagnostic and Statistical Manual for Mental Disorders (4th edn) (DSM-IV). APA, 1994.

22 First MB, Spitzer RL, Gibbon M, Williams JBW. Structured Clinical Interview for DSM-IV Axis I Disorders, Patient Edition (SCID-I/P). Biometrics Research, New York Psychiatric Institute, 1995

23 Velakoulis D, Wood SJ, Wong MT, McGorry PD, Yung A, Phillips L, et al. Hippocampal and amygdala volumes according to psychosis stage and diagnosis: a magnetic resonance imaging study of chronic schizophrenia, first-episode psychosis, and ultra-high-risk individuals. Arch Gen Psychiatry 2006; 63: 139-49.

24 Szeszko PR, Robinson DG, Sevy S, Kumra S, Rupp Cl, Betensky JD, et al. Anterior cingulate grey-matter deficits and cannabis use in first-episode schizophrenia. Br J Psychiatry 2007; 190: 230-6.

25 Yücel M, Stuart GW, Maruff P, Velakoulis D, Crowe SF, Savage G, et al. Hemispheric and gender-related differences in the gross morphology of the anterior cingulate/paracingulate cortex in normal volunteers: an MRI morphometric study. Cereb Cortex 2001; 11: 17-25.

26 Vogt BA, Nimchinsky EA, Vogt $\sqcup$, Hof PR. Human cingulate cortex: surface features, flat maps, and cytoarchitecture. J Comp Neurol 1995; 359 : 490-506.

27 Dale AM, Fischl B, Sereno MI. Cortical surface-based analysis I. Segmentation and surface reconstruction. Neuroimage 1999; 9: 179-94.

28 Fischl B, Dale AM. Measuring the thickness of the human cerebral cortex from magnetic resonance images. Proc Natl Acad Sci USA 2000; 97: 11050-5.

29 Han X, Jovicich J, Salat D, van der Kouwe A, Quinn B, Czanner S, et al. Reliability of MRI-derived measurements of human cerebral cortical thickness: the effects of field strength, scanner upgrade and manufacturer. Neuroimage 2006; 32: 180-94.

30 Rosas HD, Liu AK, Hersch S, Glessner M, Ferrante RJ, Salat DH, et al. Regiona and progressive thinning of the cortical ribbon in Huntington's disease. Neurology 2002; 58: 695-701
31 Narr K, Thompson P, Sharma T, Moussai J, Zoumalan C, Rayman J, et al. Three-dimensional mapping of gyral shape and cortical surface asymmetries in schizophrenia: gender effects. Am J Psychiatry 2001; 158: 244-55.

32 Goldstein JM, Seidman L, O'Brien LM, Horton NJ, Kennedy DN, Makris N, et al. Impact of normal sexual dimorphisms on sex differences in structural brain abnormalities in schizophrenia assessed by magnetic resonance imaging. Arch Gen Psychiatry 2002; 59: 154-64.

33 Free SL, Bergin PS, Fish DR, Cook MJ, Shorvon SD, Stevens JM. Methods for normalization of hippocampal volumes measured with MR. AJNR Am J Neuroradiol 1995; 16: 637-43.

34 Rakic P. Specification of cerebral cortical areas. Science 1988; 241: 170-6.

35 Sassi RB, Brambilla P, Hatch JP, Nicoletti MA, Mallinger AG, Frank E, et al. Reduced left anterior cingulate volumes in untreated bipolar patients. Biol Psychiatry 2004; 56: 467-75.

36 Kaur S, Sassi RB, Axelson D, Nicoletti M, Brambilla P, Monkul ES, et al. Cingulate cortex anatomical abnormalities in children and adolescents with bipolar disorder. Am J Psychiatry 2005; 162: 1637-43.

37 Adler CM, Levine AD, DelBello MP, Strakowski SM. Changes in gray matter volume in patients with bipolar disorder. Biol Psychiatry 2005; 58: 151-7.

38 Glahn DC, Bearden CE, Barguil M, Barrett J, Reichenberg A, Bowden CL, et al. The neurocognitive signature of psychotic bipolar disorder. Biol Psychiatry 2007: 62: 910-6.

39 Sesack SR, Deutch AY, Roth RH, Bunney BS. Topographical organization of the efferent projections of the medial prefrontal cortex in the rat: an anterograde tract-tracing study with Phaseolus vulgaris leucoagglutinin. J Comp Neurol 1989; 290: 213-42.

40 Sullivan RM, Gratton A. Lateralized effects of medial prefrontal cortex lesions on neuroendocrine and autonomic stress responses in rats. J Neurosci 1999; 19: $2834-40$.

41 Ryan MC, Collins $\mathrm{P}$, Thakore $\mathrm{JH}$. Impaired fasting glucose tolerance in firstepisode, drug-naive patients with schizophrenia. Am J Psychiatry 2003; 160 284-9.

42 Pariante CM, Dazzan P, Danese A, Morgan KD, Brudaglio F, Morgan C, et al. Increased pituitary volume in antipsychotic-free and antipsychotic-treated patients of the AESOP first-onset psychosis study. Neuropsychopharmacology 2005; 30: 1923-31.

43 Houenou J, Wessa M, Douaud G, Leboyer M, Chanraud S, Perrin M, et al. Increased white matter connectivity in euthymic bipolar patients: diffusion tensor tractography between the subgenual cingulate and the amygdalohippocampal complex. Mol Psychiatry 2007: 12: 1001-10.

44 Axelson DA, Doraiswamy PM, Boyko OB, Rodrigo Escalona P, McDonald WM, Ritchie JC, et al. In vivo assessment of pituitary volume with magnetic resonance imaging and systematic stereology: relationship to dexamethasone suppression test results in patients. Psychiatry Res 1992; 44: 63-70.

45 Garner B, Pariante CM, Wood SJ, Velakoulis D, Phillips L, Soulsby B, et al. Pituitary volume predicts future transition to psychosis in individuals at ultrahigh risk of developing psychosis. Biol Psychiatry 2005; 58: 417-23.

46 MacLullich AM, Ferguson KJ, Wardlaw JM, Starr JM, Deary IJ, SeckI JR. Smaller left anterior cingulate cortex volumes are associated with impaired hypothalamic-pituitary-adrenal axis regulation in healthy elderly men. J Clin Endocrinol Metab 2006; 91: 1591-4.

47 Cerqueira JJ, Catania C, Sotiropoulos I, Schubert M, Kalisch R, Almeida OF, et al. Corticosteroid status influences the volume of the rat cingulate cortex $-a$ magnetic resonance imaging study. J Psychiatr Res 2005; 39: 451-60.

48 Kajantie E, Phillips DI. The effects of sex and hormonal status on the physiological response to acute psychosocial stress. Psychoneuroendocrinology 2006; 31: 151-78.

49 Lappin JM, Morgan K, Morgan C, Hutchison G, Chitnis X, Suckling J, et al. Gray matter abnormalities associated with duration of untreated psychosis. Schizophr Res 2006; 83: 145-53.

50 Dazzan P, Morgan KD, Orr K, Hutchinson G, Chitnis X, Suckling J, et al. Different effects of typical and atypical antipsychotics on grey matter in first episode psychosis: the AESOP study. Neuropsychopharmacology 2005; $\mathbf{3 0}$ 765-74.

51 McCormick L, Decker L, Nopoulos P, Ho BC, Andreasen N. Effects of atypical and typical neuroleptics on anterior cingulate volume in schizophrenia. Schizophr Res 2005; 80: 73-84.

52 Nelson HE, Wilson JR. National Adult Reading Test, Second Edition: Test Manual. nferNelson, 1991.

53 van der Gaag M, Hoffman T, Remijsen M, Hijman R, de Haan L, van Meijel B, et al. The five-factor model of the Positive and Negative Syndrome Scale II: a ten-fold cross-validation of a revised model. Schizophr Res 2006; 85: 280-7. 